\title{
Erratum to: Long-term clinical outcomes in type 1 Gaucher disease following 10 years of imiglucerase treatment
}

\author{
Neal J. Weinreb • Jack Goldblatt • Jacobo Villalobos • \\ Joel Charrow • J. Alexander Cole • Marcelo Kerstenetzky • \\ Stephan vom Dahl • Carla Hollak
}

Published online: 28 August 2013

(C) SSIEM and Springer Science+Business Media Dordrecht 2013

Erratum to: J Inherit Metab Dis (2013) 36:543-553
DOI 10.1007/s10545-012-9528-4

Figure 2 (file name: 10545_2012_9528_Fig2_ESM.jpg) in the Supplementary material of this paper contains an error: A percentage in part A of the image was given incorrectly: 37 from 87 is $42.5 \%$ not $52.5 \%$.

Communicated by: Gregory M. Pastores

The online version of the original article can be found at http://dx.doi.org/ 10.1007/s10545-012-9528-4.

Electronic supplementary material The online version of this article (doi:10.1007/s10545-013-9648-5) contains supplementary material, which is available to authorized users.

N. J. Weinreb $(\square)$

University Research Foundation for Lysosomal Storage Diseases, Inc, Northwest Oncology Hematology Associates PA, 8170 Royal Palm Boulevard, Coral Springs, FL 33065, USA

e-mail: boneal@winning.com

J. Goldblatt

Genetic Services \& Familial Cancer Program of Western Australia,

School of Pediatrics and Child Health, University of Western

Australia, Perth, WA, Australia

J. Villalobos

Universidad Central de Venezuela, Caracas, Venezuela

J. Charrow

Division of Genetics, Birth Defects and Metabolism, Ann and

Robert H. Lurie Children's Hospital of Chicago, Chicago, IL, USA
J. A. Cole

Genzyme, a Sanofi company, Cambridge, MA, USA

M. Kerstenetzky

Instituto de Medicina Integral Professor Fernando Figueira, Recife, Pernambuco, Brazil

S. vom Dahl

Klinik für Innere Medizin, St. Franziskus-Hospital, Cologne, Germany

C. Hollak

Department of Internal Medicine, Division of Endocrinology and Metabolism, Academic Medical Center, University of Amsterdam, Amsterdam, The Netherlands 